\title{
Review of: "Hepatitis B virus infection in the Lao PDR: A systematic review"
}

Jonas Michel Wolf

Potential competing interests: The author(s) declared that no potential competing interests exist.

The present manuscript is interesting because it systematically reviews the epidemiology of hepatitis $B$ in an Asian country endemic for this disease. The results shown are useful to improve epidemiological knowledge in this area. However, I question and suggest a few points:

- Why was gray literature not investigated?

- Why did only one researcher do the identification and extraction of data? Wouldn't it be ideal for two or three independent researchers to carry out these activities to avoid bias?

- A statistical analysis of the quality of manuscripts selected for review may be important, such as heterogeneity analysis and funnel plot. 